\title{
Frictional weakening and slip complexity in earthquake faults
}

\author{
Bruce E. Shaw \\ Lamont-Doherty Earth Observatory, Columbia University, Palisades, New York
}

\begin{abstract}
Previous work has shown that velocity-weakening friction produces slip complexity in simple dynamical models of earthquake faults (Carlson and Langer, 1989). Here I show that a different type of dynamical instability, caused by slipweakening friction, also produces slip complexity. The deterministically chaotic slip complexity produced by slip-weakening friction in a simple one dimensional model is studied and the scaling of the distribution of sizes of events with the parameters in the model examined. In addition, a possible physical origin of frictional weakening. is examined, through a very simplified mathematical representation of a physical process proposed by Sibson (1973), whereby frictional heating causes an increase in pore fluid temperature and pressure, thereby reducing the effective normal stress and friction. The two different types of frictional weakening are derived from two opposing limits, with slip weakening occurring when the dissipation of heating is slow compared to the rupture timescale, as Lachenbruch (1980) has shown, while velocity weakening is shown to occur when the dissipation is fast compared to the rupture timescale. Since both end-member cases of frictional weakening are seen to produce slip complexity, slip complexity is argued to be a generic feature of frictional weakening and elastodynamics on a fault.
\end{abstract}

\section{Introduction}

To construct a mathematical model of earthquakes, there are two elements that must be specified: the equations of motion of the bulk and the boundary conditions on faults. Regarding the bulk equations, if one is not concerned with the propagation of radiated seismic waves but rather only with the generation of source motions, then the partial differential equations of linear elasticity should be sufficient for nearly all of the behavior displayed by earthquakes. Unfortunately, solving three-dimensional dynamic elasticity is, at this point, too expensive numerically to run many events on generally available computers. Thus researchers end up neglecting one aspect or another of fully dynamic three-dimensional elasticity, sometimes neglecting inertial terms, sometimes working in reduced spatial dimensions, sometimes treating modes of rupture which simplify the tensorial field to a scalar field, and very often using more than one of these simplifications. Opinions differ as to how relevant the results obtained with these various simplifications are for the full case of interest. Nevertheless, there is, at least, little disagreement over what the bulk equations of the full case are, and, with the inexorable improvements in computers, we will no doubt be able to simulate the full case in the not too distant future.

Copyright 1995 by the American Geophysical Union.

Paper number 95JB01306.

0148-0227/95/95JB-01306\$05.00
The situation is very different regarding the other element, the boundary conditions. Here there is little unanimity, and widely different approaches and opinions. Specifying the boundary conditions on faults involves specifying the geometry of the faults and the constitutive equations that determine stresses on the fault, as functions of present motions, previous motions, and other factors. One way to separate the variety of effects is to note that because of the small strain changes involved in earthquakes (less than $10^{-3}$ ) geometrical and material irregularities can be treated as being essentially fixed over many earthquake cycles. In contrast, constitutive equations which depend on motions can change substantially over the course of an individual event. Which of these two features of the boundary conditions, let's call them fixed versus dynamic, may be underlying which aspects of the many complex features displayed by earthquakes is currently an open and heated debate. Most likely the impact of fixed irregularities will be displayed in many ways in earthquake behavior. A big problem, however, is that it is very difficult to quantify the distributions of material and geometrical irregularities and relate them to geological observations, and therefore difficult to constrain the forward modeling. And, even given a distribution of fixed irregularities, one still has to specify some kind of dynamic changes during an event to determine how the system evolves. One approach has been, instead, to ask what behavior might the purely dynamic effects induce; in particular, suppose one took the limiting case where there were no fixed irregularities at all: what are the consequences of the dynamic constitutive equations on a. spatially uniform fault? 
Having reduced the issue of boundary conditions to just the dynamic constitutive equations, the obvious question is what are they; or, collapsing all of the features of surfaces in contact (fracture energies, dissipation during sliding, and so on) into the word "friction," we can better ask the question: what are the equations for friction on an earthquake fault? And what are some of the consequences of these equations?

Answering the question of what the friction is on earthquake faults is neither an easy nor straightforward task. A number of things complicate the issue, including the presence of pore fluids [Sibson, 1973], the mechanical and chemical healing that takes place between events [Dieterich, 1978], the high slip rates that events occur at, and possible opening modes [Brune, et al., 1993], not to mention the large temperatures, pressures, and scales at which the events are occurring. Fortunately, however, we can still make progress on the question of what the consequences of any particular friction might be, even without certainty that any particular form is the one happening in the Earth. By postulating a functional form and studying the consequences of that form, we can discuss what types of behavior are generic to different classes of functions, as well as what types of behavior are specific. The arbiter then becomes a check of consistency of the behavior for a particular class of functions, with the behavior seen in the Earth.

Previous work has shown that velocity-weakening friction produces slip complexity in a simple dynamical model of an earthquake fault [Carlson and Langer, 1989]. In this paper, I show that slip-weakening friction also produces slip complexity in the simple dynamical model. Thus slip complexity is argued to be a generic consequence of frictional weakening and elastodynamics on a fault.

While the result that slip-weakening friction produces slip complexity stands independently of any particular origin of the friction, the paper also contains a discussion of a physical example which shows how frictional weakening might arise in the Earth. The physical process is one proposed by Sibson [1973], whereby frictional heating raises the temperature and pressure of pore fluids, thereby decreasing the normal stress and friction. I derive velocity weakening and slip weakening from two opposing limits of a simplified mathematical representation of this physical process. In the limit where heat dissipation is slow compared to the rupture timescale, slip weakening results, as Lachenbruch [1980] has shown, while in the limit where heat dissipation is fast compared to the rupture timescale, velocity weakening results. Dissipation of pore pressure by pore volume expansion would suggest that the dissipation timescale is more of the order of the rupture timescale, so that the relevant limit for the Earth would be intermediate between these two extremes. Nevertheless, the slip-weakening limit is of interest as an end-member case, as well as being a result of a variety of other physical mechanisms.

This grounding of the discussion of frictional weakening also leads us to a consideration of another physical effect Sibson [1973] discusses which might be relevant in limiting the drop in friction, and that is hydrofracturing. The qualitative features of the distribution of sizes of events in the model is shown to be relatively insensitive to the nonlinear form of the saturation of the friction drop; the results are thus shown to be insensitive to the way in which the drop in friction may be limited by such processes as hydrofracturing.

The paper is organized as follows. In the next section, section 2 , the equations representing the physical effects of frictional heating are presented, and, in two opposite limits, the resulting functional forms of frictional weakening are derived. In section 3 , the equations for the uniform one-dimensional dynamical model which will be solved are presented. A minimal parameterization of the equations is given. In section 4 , results of the numerical simulations of the equations are shown, which show the nonperiodic complex distributions of slip which develop, examine the dependence of the distributions of sizes of events on the various parameters, note some differences between the slip-weakening and velocity-weakening complexity, and show the relative insensitivity of the results to the exact functional form of the frictional weakening. The paper concludes in section 5 with a discussion and summary of the results.

\section{Derivation of Frictional Weakening from Frictional Heating}

Friction has been recognized for a number of decades as the key ingredient that causes the slow buildup of tectonic motions to be released in the sudden stick-slip events we experience as earthquakes [Brace and Byerlee, 1966]. In an effort to develop functional forms that would describe the friction operating on earthquake faults, a number of experiments involving the controlled sliding of rocks and other materials have been carried out, at a variety of temperatures and pressures [Scholz et al., 1972; Dieterich, 1978]. Among the successes of these experiments was a good match between the temperatures and pressures where frictional weakening versus frictional strengthening effects were seen, implying unstable versus stable sliding, and the corresponding depths where earthquakes as opposed to stable sliding are observed to occur [Brace and Byerlee, 1970; Stesky et al., 1974; Tse and Rice, 1986; Blanpied et al., 1991]. Another success was the wide variety of materials that showed very similar behavior in the experiments, and the resulting wide applicability of the functional forms that were developed to describe this behavior [Dieterich, 1979; Ruina, 1983]. A basic, open question, however, is whether these functional forms could be simply extrapolated to apply to the higher slip rates and geological settings of earthquakes, and whether other physical processes might need to be considered. One clue that significant modifications might be required came from simple considerations of the heat that should be generated during earthquakes. With standard representations of typical friction behavior, rocks on the fault should melt [McKenzie and Brune, 1972]. While 
this was observed to occur sometimes, in general, it was not [Sibson, 1975]. Another related observation was the lack of heat flow at the surface near faults that would be expected to be observed given usual frictional heating [Brune, et al., 1969; Lachenbruch and Sass, 1980]. Quite some time ago, Sibson [1973] had offered one possible explanation of the paradoxes posed by heating, and that was that the frictional heating raised the temperature and pressure of pore fluids, water, which were present along faults, and that the raised pressure reduced the effective normal stress and thus reduced the friction. Sibson also noted that another physical effect might be relevant in cutting off the reduction in friction by pore pressure, and that is that hydrofracturing can occur whereby tensile cracks open when the pore pressure exceeds the least principal stress [Sibson, 1973]. In this section, I examine very simple mathematical representations of these physical processes and examine the frictional weakening that arises from frictional heating.

Friction $\Phi$ is given by the product of the normal force $N$ times a coefficient of friction $\mu$ :

$$
\Phi=N \mu
$$

We will take the simplest kind of stick-slip coefficient, the kind taught in high school physics, which resists sliding up to some maximum value $\mu_{0}$ when it is stuck, and has a constant, smaller value $\mu_{0}(1-\sigma)$ when it is sliding:

$$
\mu= \begin{cases}{\left[-\mu_{0}, \mu_{0}\right]} & \frac{\partial S}{\partial t}=0 ; \\ -\mu_{0}(1-\sigma), \mu_{0}(1-\sigma) & \frac{\partial S}{\partial t}<0, \frac{\partial S}{\partial t}>0 .\end{cases}
$$

Here, $\frac{\partial S}{\partial t}$ is the slip rate, which is the change in slip $S$ with respect to time $t$. When sticking, friction adjusts to resist motion, up to a maximum size, so $\mu$ is multivalued when $\frac{\partial S}{\partial t}=0$. When sliding, the sign of $\mu$ changes when the sign of $\frac{\partial S}{\partial t}$ changes since friction resists sliding. This simplified form of $\mu$ neglects all of the complications that have been learned about how rate and state dependences enter in as $\mu$ changes from sticking to slipping. Here we neglect all those effects which the standard rate and state dependent constitutive laws have been developed to describe [Dieterich, 1979] and instead collapse them all into a single simple drop to the sliding value. There are two reasons for doing this. The main reason is that we will be concerned with feedback that affects the friction by changing the effective normal force, not the friction coefficient, and this change in the normal force can be large compared to the change in the friction coefficient. A second reason is that in the standard constitutive laws, the rate dependence is typically logarithmically dependent on the rate, and thus most of the drop occurs at very low velocities, and the slip distance over which this change occurs is very small compared to typical earthquake slip distances [Dieterich, 1979]. Thus, at even a small fraction of the scales that earthquakes occur at, a full drop in the friction coefficient will have occurred. We will be interested in changes that happen more on the scale that earthquakes occur, and, as will be seen, they can be quite substantial. Other effects on $\mu$ at higher velocities have also been described [Blanpied, et al., 1987]. While all the subtleties of the changes in $\mu$ could be incorporated into the analysis numerically, the point here is to show that there can be large changes in the normal stress which can dominate the frictional evolution, which we can gain some insight into analytically by neglecting the subtle changes in $\mu$.

The next equation is the key point, and that is that there can be processes through which heat $Q$ can reduce the effective normal stress:

$$
N=N_{0}-\alpha Q,
$$

where $N_{0}$ is some initial ambient normal stress, and $\alpha$ is a proportionality constant which relates how strongly changes in the heat $Q$ reduce the effective normal stress. The simplest physical picture of this, for the case of earthquakes, is to consider the role of pore fluids, which are generally believed to be present in earthquake faults and play a significant role there. Increasing the heat raises the temperature, which increases the pressure [Sibson, 1973]. Equation (3) with pore pressure replacing the heat term is a standard representation of the effect of fluids.

The last equation describes how the heat changes in time $t$ :

$$
\frac{\partial Q}{\partial t}=-\gamma Q+\Phi \frac{\partial S}{\partial t}
$$

The first term on the right hand side says that heat is dissipated on a timescale of $1 / \gamma$. The second term is the rate of heat generated by frictional sliding. The dissipation of heat occurs as heat is lost from the fault zone to the surrounding media. We don't care exactly how that happens; what turns out to be most important, as we will see below, is the timescale over which the loss occurs.

\section{Case 1: Fast Heat Dissipation Gives Velocity Weakening}

In the limit when the heat dissipation timescale is short compared to the rupture time of an event, which we set to be unity, that is, when $1 \ll \gamma$, we can solve (1)(4) as follows. In this limit, we can neglect $\frac{\partial Q}{\partial t}$ compared to $\gamma Q$ in (4), giving $Q=\frac{1}{\gamma} \Phi \frac{\partial S}{\partial t}$. Substituting this into (3) and using (1) to substitute $\Phi$ for $N$ give an equation for $\Phi$ in terms of $\frac{\partial S}{\partial t}$. This can be solved to give

$$
\Phi=\frac{N_{0} \mu}{1+\frac{\alpha}{\gamma} \mu \frac{\partial S}{\partial t}}
$$

There are a few things to note about this equation. First, in the absence of any heating effect, when $\alpha=0$, we recover the usual friction. Second, it has the appropriate symmetries (recall how $\mu$ behaves) when sliding, that $\Phi\left(\frac{\partial S}{\partial t}\right)=-\Phi\left(-\frac{\partial S}{\partial t}\right)$, and when sticking. Most importantly, it shows velocity weakening, with a linear decrease in friction with slip rate at low slip rates, saturating to zero friction at high rates. 
The saturation to zero friction will likely not, however, occur on earthquake faults. Another physical effect will have kicked in before the fluid pressure completely cancels out the normal stress, and that is hydrofracturing, whereby tension cracks off of the fault surface are opened and fluidized material injected into them [Sibson, 1973]. This process initiates when the pore fluid pressure exceeds a certain fraction of the normal stress, with the fraction depending on a number of factors, including the tensile strength of the cracks relative to the overall normal stress, and the mode of rupture [Sibson, 1981]. For now, it is enough to simply acknowledge that there will in fact be a finite limit to the reduction in friction, so that completely frictionless sliding cannot occur. This issue will come up again later, and will be discussed further.

\section{Case 2: Slow Heat Dissipation Gives Slip Weak- ening}

Equations (1)-(4) can also be solved in the limit where heat is dissipated on a timescale that is long compared to the event time, which is of order 1 , though short compared to the interevent time, call it $1 / \epsilon$, with $\epsilon \ll 1$. When $\epsilon \ll \gamma \ll 1$, so that heat builds up during an event but decays completely between events, (4) is solved by neglecting $\gamma Q$ compared to $\frac{\partial Q}{\partial t}$, so $Q=\int \Phi \frac{\partial S}{\partial t} d t=\int_{S_{0}}^{S} \Phi d S$, where $S_{0}$ is the initial value of the slip at the beginning of the event. Substituting this into (3) and (1) into (3) gives an integral equation for $\Phi$. This can be solved and, when slip occurs only in one direction (positive or negative), gives

$$
\Phi=N_{0} \mu e^{-\alpha \mu\left(S-S_{0}\right)} .
$$

This exponential form for slip weakening when heat dissipation is slow compared to the rupture timescale has been obtained previously by Lachenbruch [1980]. Again, as in the previous case, the $\alpha=0$ limit and the appropriate symmetries are present in this expression. Here, though, we see slip weakening, with a linear decrease with slip at low slip. At high slip the expression shows the friction saturating to zero friction, but, as noted in the previous velocity-weakening case; hydrofracturing will have set in before this limit is reached. Friction will thus be limited to dropping to some finite fraction of its initial value. We will return to this point in the next section, when we discuss the dynamical implications of the slip-weakening friction.

\section{Case 3: Heat Dissipation on Rupture Timescale}

Aside from hydrofracturing, we have not said much about how the heat and pore pressure might be dissipated. One potentially important physical process for dissipating pore pressure is dilatancy, which changes the pore volume [Mase and Smith, 1987]. These pore volume changes could occur on the timescale that slip motions are occurring on the fault, so $\gamma$ of order unity would be the relevant dissipation timescale. This situation is intermediate between the two extreme cases we have solved above and thus involves some mixture of slip- and velocity-weakening effects. While this intermediate value can be studied numerically, we do not have a closed analytical solution. Its behavior is, however, presumably bracketed by the slip- and velocity-weakening cases; in this paper we will focus our attention on the slip-weakening case, since velocity weakening has been studied extensively before, since it is an end-member case here, and because of its relevance to other physical mechanisms of frictional weakening.

\section{Equations of Motion and Minimal Parametrization}

A new way of understanding earthquake complexity was initiated by Carlson and Langer [1989] with the observation that homogeneous deterministic models could produce complex sequences of events through inertial dynamics and a frictional instability. They studied the one-dimensional uniform Burridge-Knopoff model [ $B u r$ ridge and Knopoff, 1967], with a velocity-weakening friction law, and found a power law distribution of small events, along with a distribution of large events that exceeded the rate that would be expected by extrapolating the small event distribution, and which dominated the net motion forward in the system. Horowitz and Ruina [1989] also observed the development of complex aperiodic slip modes, in their case in a two-dimensional quasi-static model using standard rate and state dependent friction laws, though they were limited in the time they could run the model and thus could not examine statistical measures of the resulting complexity. Subsequent work on the one-dimensional Burridge-Knopoff model with velocity weakening studied a variety of aspects of the behavior, including the cycle of small event activity preceding large events [Shaw, et al., 1992], the moment source spectra [Shaw, 1993], the propagation of pulses of slip [Langer and Tang, 1991], and the long and intermediate term forecasting of large events in the model [Pepke, et al., 1994]. By coincidence, the functional form of frictional weakening used, chosen for its mathematical simplicity, happens to be essentially the same form as was derived in section 2 for the velocityweakening case, (5). Thus we already know a lot about the case of velocity-weakening, most importantly, that it gives slip complexity.

What about the slip-weakening case? Below, I present some initial results from slip-weakening friction, showing that it too produces slip complexity.

The model consists of a partial differential equation representing the time evolution of slip $S$, subject to elastic forces and a dissipative frictional force along thie fault. For reasons of computational efficiency, we will restrict ourselves here to a one-dimensional elastic media. The equations are then

$$
\frac{\partial^{2} S}{\partial^{2} t}=\frac{\partial^{2} S}{\partial x^{2}}-S+\nu t-\Phi,
$$

where $t$ is time, $x$ is distance along the fault, $\nu$ is the slow loading rate, and $\Phi$ is friction. This is Newton's 
equation in dimensionless form, saying that the acceleration of the slip is the sum of three forces: a compressional stress gradient $\partial^{2} S / \partial x^{2}$, a shear stress $\nu t-S$, and friction. Space and time have been scaled to reduce the equations to the minimum number of dynamically relevant parameters. All the nonlinearity is contained in the friction.

Since the velocity-weakening case has been extensively studied, we consider here the slip-weakening case. We need a functional form of the friction which takes into account the way that friction is limited to dropping to a finite nonzero value, here, with our frictional heating picture, because of hydrofracturing. It is easy to see that this effect is important, since if friction did drop to zero, and recovered to a nonzero value only very slowly as the heat dissipated on a timescale which was long compared to the rupture timescale, then a fault, having ruptured, would continue sliding back and forth, back and forth, as a slowly decaying oscillator. This clearly is not seen in earthquakes. Most likely, no backslip is occurring at all, and events move in only one direction.

To take into account the hydrofracturing limitation on $\Phi$, we can modify (6) to read:

$$
\Phi= \begin{cases}N_{0} \mu e^{-\alpha \mu\left(S-S_{0}\right)}, & e^{-\alpha \mu\left(S-S_{0}\right)}>h ; \\ N_{0} \mu h, & e^{-\alpha \mu\left(S-S_{0}\right)} \leq h .\end{cases}
$$

where $0 \leq h<1$ is a constant which reflects the threshold where hydrofracturing initiates. As mentioned earlier, its value will depend on a number of factors, including the tensile strength of the surrounding cracks relative to the normal stress and the fracture mode of the fault [Sibson, 1981]. However, as we will see below, its actual value is unimportant, as long as it is not too small.

We modify this frictional form further, with two considerations: ignorance of the hydrofracturing transition and a desire for a minimal parametrization of the friction. Regarding the first point, we really don't know the exact form of how the hydrofracturing comes in, and the sudden onset we have introduced may very well be smoothed somehow in a real system. Also, when we study the complexity that comes from these functions, we want to be clear that it is not due to any sharp features in the sliding regime of the friction. It is better, then, to think of classes of functions which connect an initially linear decreasing function of slip to one which asymptotically reaches some finite value. Two examples of smoothed functions which do this are the polynomial and exponential ones we encountered earlier:

$$
\Phi=N_{0} \mu \frac{1}{1+h}\left(\frac{1}{1+\alpha \mu\left(S-S_{0}\right)(1+h)}+h\right)
$$

and

$$
\Phi=N_{0} \mu \frac{1}{1+h}\left(e^{-\alpha \mu\left(S-S_{0}\right)(1+h)}+h\right) .
$$

A third, nonsmooth function, is the piecewise linear $\Phi$ :

$$
\Phi= \begin{cases}N_{0} \mu\left(1-\alpha \mu\left(S-S_{0}\right)\right), & 1-\alpha \mu\left(S-S_{0}\right)>h \\ N_{0} \mu h, & 1-\alpha \mu\left(S-S_{0}\right) \leq h .\end{cases}
$$

All of these differ from (8) only in the nonlinear way in which the initial linear decrease of the friction with slip from a value of $N_{0} \mu$, having slope $-N_{0} \mu^{2} \alpha$, matches the final friction value of $N_{0} \mu h$. In the end, we'll show numerically that the form of the nonlinear interpolation is unimportant, that all three forms give qualitatively similar results, and thus that the details of the hydrofracturing process are, fortunately, unimportant.

The second consideration is that we'd like a representation of the friction with the minimum number of parameters, so that we can most clearly see what things depend on. Here we use the lack of backslip, along with two symmetries of the dynamical equation (7), to reduce the number of parameters in the friction and see just the minimum relevant ones. The two symmetries are, first, that subtracting a constant from $\Phi$ and adding that same constant to $S$ leaves the equation invariant and, second, that multiplying $\Phi, S$, and $\nu$ all by a constant leaves the equation invariant. The first symmetry is a reflection of the way in which only stress drops, and not absolute stresses, are dynamically relevant. Working with (9), let's use the symmetries. Without loss of generality, we can restrict ourselves to $\nu>0$. By subtracting off the minimum sliding value of friction, $N_{0} \mu_{0}(1-\sigma) h$, multiplying by $\frac{1+h}{N_{0} \mu_{0}}$ to normalize the drop in friction to 1 , rescaling $S$ and $\nu$ by that same factor, and rescaling $\alpha$ by $\frac{1}{N_{0}(1-\sigma)^{2}}$ (the extra factor of $\frac{1}{1-\sigma}$ is put in so that the initial slope will be $\alpha$, not $\alpha(1-\sigma))$, we can write

$$
\Phi= \begin{cases}{\left[\frac{-1}{1+\frac{\alpha}{1-\sigma}\left(S-S_{0}\right)}-2 c, \frac{1}{1+\frac{\alpha}{1-\sigma}\left(S-S_{0}\right)}\right],} & \frac{\partial S}{\partial t}=0 ; \\ \frac{1-\sigma}{1+\frac{\alpha}{1-\sigma}\left(S-S_{0}\right)}, & \frac{\partial S}{\partial t}>0 .\end{cases}
$$

where $c=\frac{h(1-\sigma)}{1+h}$. Observe that the constant $c$, and therefore $h$, does not play any role in the dynamics, though it needs to be large enough to keep the fault from backslipping even after it has slipped for a long way. We can thus drop it entirely and write

$$
\Phi= \begin{cases}\left(-\infty, \frac{1}{1+\frac{\alpha}{1-\sigma}\left(S-S_{0}\right)}\right], & \frac{\partial S}{\partial t}=0 \\ \frac{1-\sigma}{1+\frac{\alpha}{1-\sigma}\left(S-S_{0}\right)}, & \frac{\partial S}{\partial t}>0\end{cases}
$$

which reduces $\Phi$ to the minimum amount of parameters, $\sigma$ and $\alpha$. Between events, of course, all the built up heat dissipates, so $S_{0}$ is reset to its new value and $S=S_{0}$ at the beginning of each event. be

By the same steps, the exponential form of $\Phi$ would

$$
\Phi= \begin{cases}\left(-\infty, e^{-\frac{\alpha}{1-\sigma}\left(S-S_{0}\right)}\right], & \frac{\partial S}{\partial t}=0 \\ (1-\sigma) e^{-\frac{\alpha}{1-\sigma}\left(S-S_{0}\right)}, & \frac{\partial S}{\partial t}>0\end{cases}
$$

and the piecewise linear form would be 


$$
\Phi=\left\{\begin{array}{cc}
\left(-\infty, 1-\alpha\left(S-S_{0}\right)\right], & \frac{\partial S}{\partial t}=0 \text { and } \\
1-\sigma-\alpha\left(S-S_{0}\right)>0 & \frac{\partial S}{\partial t}>0 \text { and } \\
1-\sigma-\alpha\left(S-S_{0}\right), & 1-\sigma\left(S-S_{0}\right)>0 \\
& \frac{\partial S}{\partial t}=0 \text { and } \\
1-\sigma-\alpha\left(S-S_{0}\right) \leq 0 & \frac{\partial S}{\partial t}>0 \text { and } \\
1-\sigma-\alpha\left(S-S_{0}\right) \leq 0
\end{array}\right.
$$

Because the previous velocity-weakening work was done with the polynomial form, we'll typically use that. Later, though, we will examine the different forms of the nonlinear interpolations and see that they seem to have only minor quantitative but no qualitative effects on the behavior. In either of the cases, the velocity-weakening friction would be the same as in (13), (14), or (15) but with $\partial S / \partial t$ replacing $S-S_{0}$ and $\alpha$ having been rescaled by an extra factor of $1 / \gamma$.

While the total forces on the fault remain less than the threshold force of 1 , the fault remains stuck with $\partial S / \partial t=0$ and is slowly loaded at rate $\nu \ll 1$. When the threshold force is exceeded, the fault begins to slide with initial acceleration $\sigma$. When $\nu \ll 1$ and $\sigma>0, \nu$ becomes irrelevant to the dynamics and only sets the timescale between events. In the Earth, where event times are of order seconds and loading times are of order hundreds of years, $\nu \sim 10^{-9}$ or less, so the separation of timescale of $\nu \rightarrow 0$ is clearly an excellent approximation. Thus we see the evolution equations, (7) with, say, (13) only depend, apparently, on two parameters, $\sigma$ and $\alpha$.

In the velocity-weakening case, however, an additional parameter was seen to enter into the problem, which came from the fact that velocity-weakening friction leads to an instability which extends to the smallest lengthscales in the problem. This manifested itself in a dependence of even the largest events on the small numerical discretization length in the pure velocityweakening case [Carlson, et al., 1991] or, when a stabilizing viscous term $\eta \frac{\partial^{3} S}{\partial t \partial x^{2}}$ was added to that dynamics [Myers and Langer, 1993; Langer and Nakanishi, 1993], in a dependence on the viscous coefficient $\eta$ [Shaw, 1994]. (Spatial discreteness is not the cause of complexity in these fully dynamic models [Shaw, 1994], unlike some quasi-static models [Rice, 1993]).

What about the slip-weakening case? A linear stability analysis of the growth of Fourier modes in the two cases is helpful in examining the issue of the relevance of small length scales in the dynamics. Linearizing the equation of motion (7), we examine the growth of a Fourier mode $e^{i k x+\Omega t}$. This gives a dispersion relation of

$$
\Omega=\frac{\alpha \pm \sqrt{\alpha^{2}-4\left(k^{2}+1\right)}}{2}
$$

in the case of velocity weakening [Carlson and Langer, 1989], and

$$
\Omega= \pm \sqrt{\alpha-1-k^{2}}
$$

in the case of slip weakening. Observe that in the case of velocity weakening all wavelengths have a positive real $\Omega$ growth rate and are thus unstable, while the the slip-weakening case, wavelengths with $k<\sqrt{\alpha-1}$ are unstable while $k \geq \sqrt{\alpha-1}$ have $\operatorname{Re} \Omega=0$ and are thus marginally stable. In the velocity-weakening case, adding some kind of term that stabilized small wavelengths, for example, the viscous term described above, was necessary to give a proper continuum limit. What about the slip-weakening case? Is it the smallest unstable wavelength that is relevant to determining whether there is a good continuum limit, in which case no additional term and parameters are necessary? Is it the smallest marginally unstable wavelength that is relevant, in which case some additional stabilizing term is again necessary and again a relevant parameter? The answer requires a study of the full nonlinear equations, which we can only do numerically. As we will see, however, it turns out that, at least for the distribution of sizes of events, the slip-weakening case does have a well-behaved continuum limit, and that no additional terms and parameters therefore need to be introduced. There is thus one fewer essential parameter in the slip-weakening friction dynamics as compared to the velocity-weakening dynamics, and in this sense, the slip-weakening case is simpler.

The last element that must be specified is the boundary conditions. To obtain a completely uniform fault, we use periodic boundary conditions. One final possible parameter is then the system size, $N$. However, as long as $\alpha$ is big enough, and the system is long enough, the largest events turn out not to span the system size, and thus $N$ is also an unimportant parameter. So the minimal number of parameters in the slip-weakening case is remarkably few, reducing to only two: $\alpha$ and $\sigma$. Further, as we will see, the dependence on $\sigma$ is particularly simple, so that really we are left with only one important parameter, $\alpha$.

Given that all of the important physical parameters have collapsed into basically one unknown constant, $\alpha$, it would be nice if we could derive what $\alpha$ should be, given physical constants of the Earth. Unfortunately, uncertainties in the physical constants and mechanisms of heat transport are so large that that approach isn't very constraining. What we can say is the following. The parameter $\alpha$ has the dimensions of an inverse distance, in units of the slip of a large event. For perfect efficiency in converting frictionally generated heat into pore fluid pressure, $\alpha$ would be given roughly by (neglecting the factor of $\mu$, which is of order unity) the ratio of the slip distance in a large event (meters) to the distance over which the heat generated is distributed over during the fast rupture timescale, a number that might be, say, centimeters over the fault gouge. Of course, not all the heat goes into increasing fluid pressure (some goes into heating the rock, for example), and a significant amount of pressure could be lost to dilatancy effects, so $\alpha$ would be only some fraction of this ratio of distances (The loss of heat to hydrofracturing is not relevant here, as $\alpha$ corresponds to the initial drop 
in friction). Nevertheless, for the scenario mentioned above, heat from meters of slip confined to a width of centimeters across the fault, values of $\alpha$ larger than unity, the large $\alpha$ regime we will end up discussing in this paper, seem quite possible (see also [Lachenbruch, 1980]).

\section{Slip Complexity in Numerical Simulations}

Having set up the equations of motion, we solve them numerically. Again, while a physical picture has been given for a possible origin of the friction that will be used, the numerical results stand independently of that origin. Thus here we address the question of the dynamics implications of slip-weakening friction.

Equations (7) and (13) are solved numerically with periodic boundary conditions, using a finite difference approximation for the spatial derivatives, and an implicit time stepping. Making a finite difference approximation of the spatial derivatives in the massive one-dimensional wave equation gives a set of coupled ordinary differential equations, which are the block and spring model introduced by Burridge and Knopoff [1967], and shown in Figure 1. The simulations were run on a SUN Sparc workstation, with tens of thousands of events being obtainable on overnight runs.

The system is loaded until a part of the fault reaches the threshold for slipping. The value of the initial slip $S_{0}$ along the fault is set then. Using the separation of timescales, $\nu \ll 1$, the loading is held fixed while an event occurs. Events begin at the epicenter with an initial acceleration of $\sigma$, and motion there may or may not trigger neighboring regions to move. Eventually the system comes to rest and an event is completed. The fault is then reloaded, $S_{0}$ is reset to the new initial starting values, and a new event begins.

Beginning from any nonsmooth initial condition, the system evolves to a statistically steady state. The system is chaotic in the sense that nearby inital condi- tions diverge exponentially from one another, on average. Two different types of behavior are displayed by the model, with the division evidenced by the dispersion relation (17). When $\alpha>1$, the long wavelengths are unstable, and the system evolves to a complex state; events do not span the system for large enough system size; there are small events that scale with $\sigma$ and large events that are independent of $\sigma$; and there is great irregularity along the fault which is intrinsic to the dynamics. When $\alpha<1$, there is only one type of event, and it scales with $\sigma$. While complex slip with finite size events that do not span the system can still develop here, this complexity is not robust, in that small amounts of stable dissipation lead to events spanning the system. So, for example, adding a stabilizing term $-\Gamma \frac{\partial S}{\partial t}$ to (7) leads to systemwide events when $\alpha$ is small and $\Gamma$ is bigger than some (small) number. Because complexity in the $\alpha<1$ regime is less robust and depends more on the details of $\mu$, we will focus our attention in this paper on the generic complexity in the $\alpha>1$ case. Focusing attention on the large $\alpha$ case is also justified by the arguments at the end of the previous section, which suggested that large values of $\alpha$ might be relevant to earthquake faults.

The complex evolution produces a wide range of sizes of events. The size of an event is given by the moment $M$, which is the sum of the net slip in an event:

$$
M=\int\left(S-S_{0}\right) d x
$$

where $S$ is measured when the fault comes to rest at the end of an event. The magnitude of an event, which we'll call $w$, is the logarithm of the moment:

$$
w=\ln M \text {. }
$$

Figure 2 shows two different representations of the slip complexity that develops. In Figure 2a, the times at which various portions of the fault become unstuck are plotted. In Figure $2 \mathrm{~b}$, the sequence of stuck con-

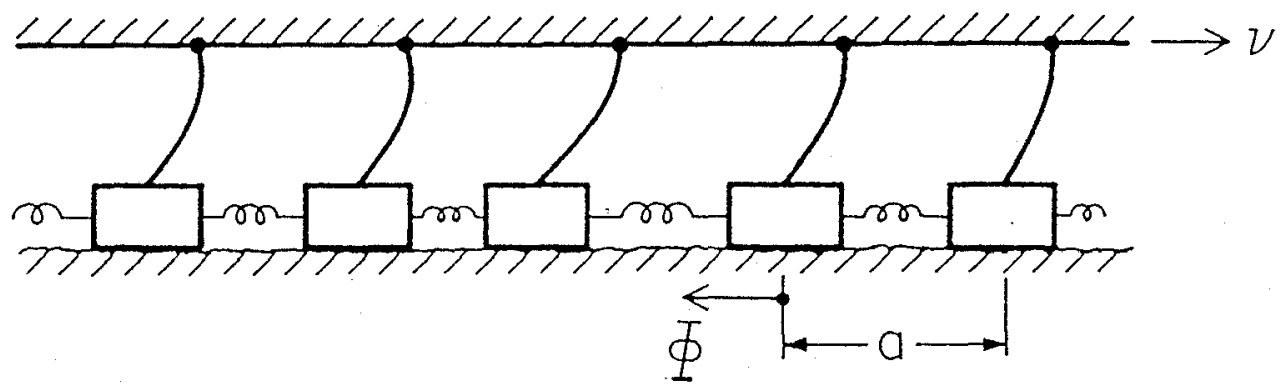

Figure 1. The finite difference approximation of the one-dimensional massive wave equation gives the block and spring model shown here. The blocks, having uniform masses, are connected to each other by a set of uniform springs, and to a plate above by another set of uniform springs. They rest on a plate below and experience a friction force $\Phi$, which resists motion, as the two plates are slowly sheared at a rate $\nu$ with respect to each other. The spacing between blocks which have slipped the same amount is the length $a$. The slip $S$ of each block is measured by the amount the block has moved with respect to the lower plate. When $\Phi$ decreases sufficiently with either slip or slip rate, the slips $S$ along the fault are seen to evolve into a rough, irregular pattern. 

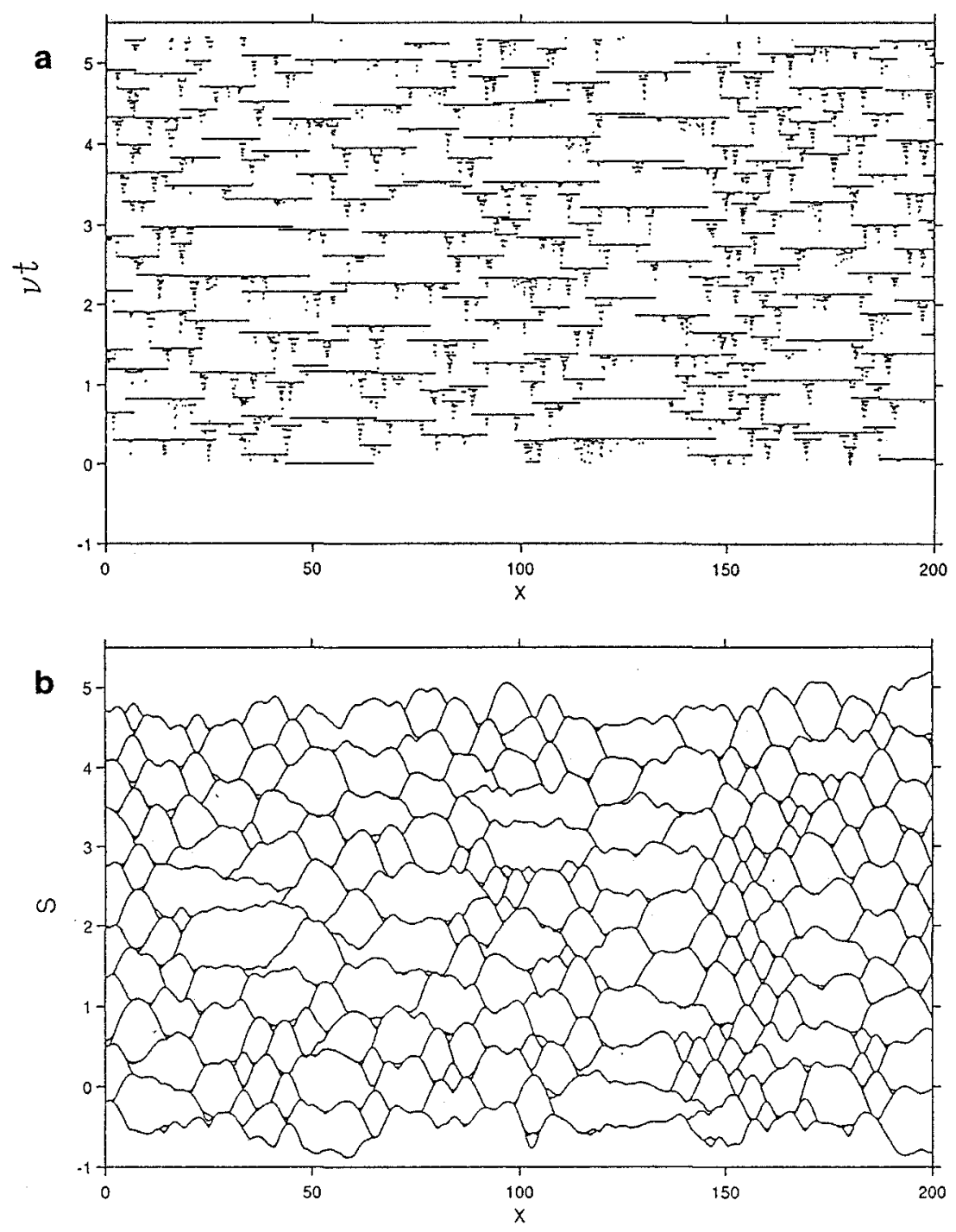

Figure 2. Two different ways of looking at the slip complexity of a typical sequence produced in the model. Figure 2 a shows the times at which various portions of the fault ruptured; this is a standard plot in seismology, but one which here, in the model, can be made for arbitrarily large sequences in time, and large uniform lengths in space. Figure $2 \mathrm{~b}$ shows a different representation of the same events shown in Figure 2a, only now the cumulative slip following each event is plotted. Here, we see that the largest events dominate the net motion forward; the numerous small events, which can be seen in Figure 2a, are difficult to see here. Note the persistence of similar large events along the fault. These features are reminiscent of "characteristic events" and segment behavior seen in real faults. The fault here is, though, completely homogeneous, so that features persist only for some time, and all parts of the fault behave the same, in the long run. The parameter values used in Figure 2 are $\alpha=4, \sigma=.1$, and $a=.1$.

figurations for the same events shown in Figure 2a is plotted. There is clearly a range of sizes of events, with many small events, and fewer large events. The large events do, however, dominate the net motion forward; the numerous small events are difficult to see in Figure $2 \mathrm{~b}$ because they are so small. This aspect of the complexity can be quantified by looking at the distribution of sizes of events.

Examining the distribution of sizes of events, we can show the unimportance of the spatial discretization length $a$, the simple dependence on $\sigma$, and the significant dependence on $\alpha$. We plot the differential rate of events $R(w)$ having magnitudes between $w$ and $w+d w$, as a function of $w$, with $R$ being the rate of events per unit displacement per unit fault length, so $\int R(w) e^{w} d w=1$. (Note that $R$ is not the cumulative distribution of events bigger than a certain magnitude, which is often the way real data are plotted because of the sparsity of the data).

Figure 3 shows a set of distributions for fixed $\alpha$ and $\sigma$ and different spatial discretization lengths $a$. In all these figures, $N$ is large and irrelevant. Note that the curves completely overlay; except for the finite cutoff that the spatial discretization imposes on the size of the 


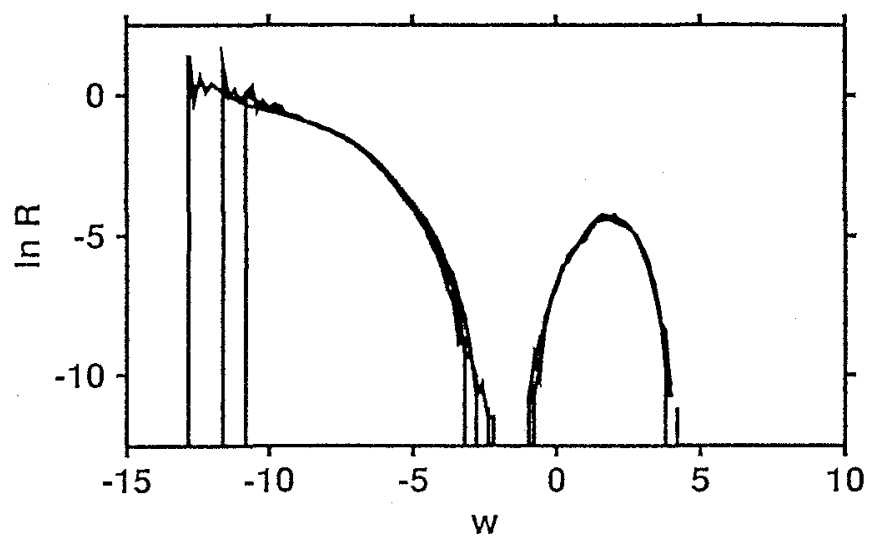

Figure 3. The distribution of sizes of events for different small length cutoffs $a$. The differential rate of events $R(w)$ having magnitudes between $w$ and $w+d w$ is shown, with the logarithm of $R$ versus the magnitude $w$, which is the logarithm of the moment $M$, being plotted. There are three curves, each of which have the same values of slip weakening $\alpha=4$ and initial friction drop $\sigma=.01$ but different small discretization lengths, with $a=.1, .075$, and .05 used. Note that, aside from setting the cutoff size of the very smallest events, all the curves collapse onto each other, so that the parameter $a$ is an irrelevant parameter in determining the behavior and that there is a well-defined continuum limit $(a \rightarrow 0)$ distribution that all the curves are collapsing onto. Because the value of $a$ is irrelevant, it will be fixed for the rest of the figures, at a constant value $a=.1$. Note that the very smallest events, those here with magnitude $w$ less than around -8 , follow a straight line on this $\ln R$ versus $w$ plot and thus have a power law distribution; this extends out arbitrarily far, depending on how small $a$ is.

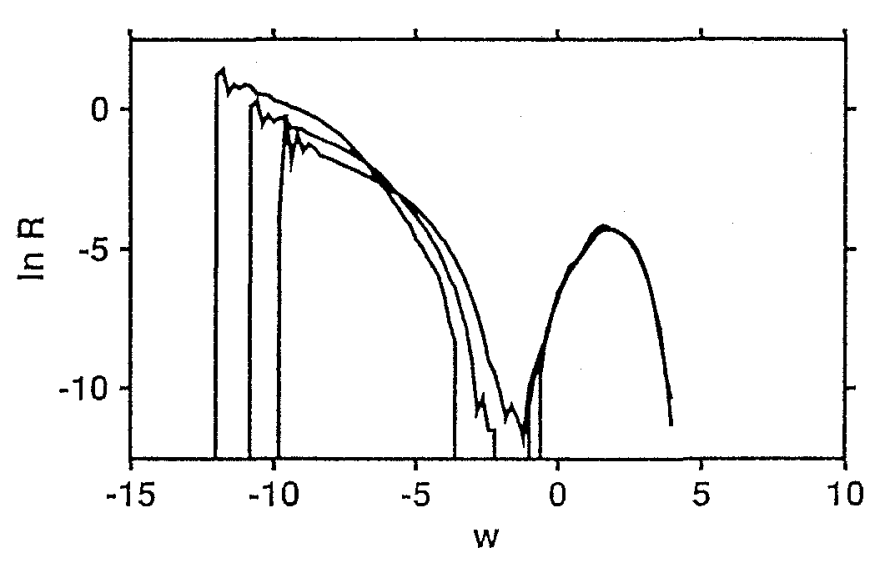

Figure 4. The distribution of sizes of events for different initial friction drops $\sigma$. The same type of plot as in Figure $3, \ln R$ versus $w$, is shown (and will be shown in a number of figures that follow). Here the three curves have the same value of $\alpha=4$ but different values of $\sigma=.03, .01$, and .003 . Note that the large event distributions are the same for each of the curves, showing the large events to be independent of $\sigma$, while the small event distributions change.

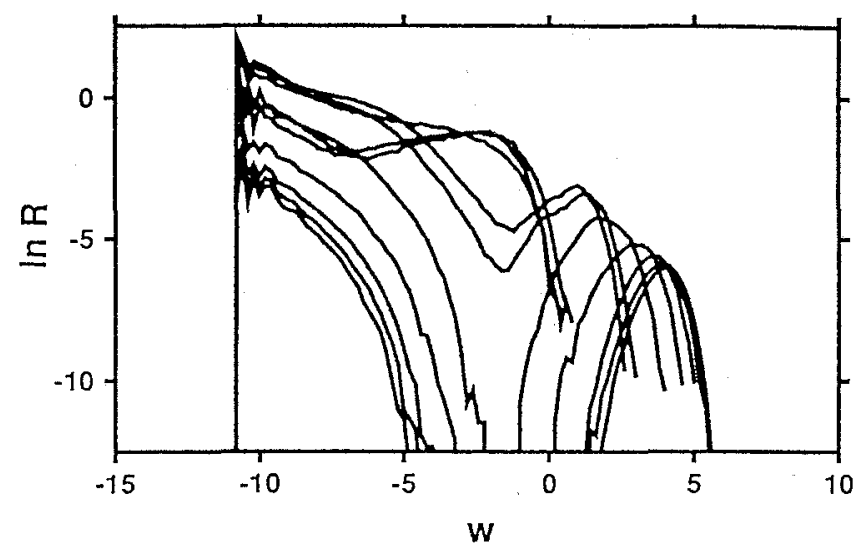

Figure 5. The dependence of the distribution of sizes on the slip-weakening parameter $\alpha$. The curves have the same value of $\sigma=.01$ but different values of $\alpha=$ $.25, .5,1,1.5,2,4,6,8,10,12$ from top to bottom, respectively. Both the small and large events are seen to depend on $\alpha$. For $\alpha \leq 1$ there are only small events, which scale with $\sigma$.

very smallest events, the curves are the same and converge to a well-defined continuum limit. Having shown the unimportance of $a$, we will fix it for the rest of the discussion.

Figure 4 shows a set of distributions where $\alpha$ is fixed, while $\sigma$ is varied. The existence of two different types of events in the model can be seen in this figure, with the small events scaling with $\sigma$, while the large events are independent of $\sigma$. The distribution of sizes of small events depends very simply on $\sigma$; a simple rescaling of the moment by $1 / \sigma$ and the rate by $\sigma$ shows the the different small event curves collapse onto each other.

The key parameter is $\alpha$. Figure 5 shows a set of distributions where $\sigma$ is fixed and $\alpha$ is varied. The dependence on $\alpha$ is clearly complicated, for both the large and small events. Before seeing if there is some scaling with $\alpha$, that may at least apply for large $\alpha$, let's look at some simple estimates of what some of the relevant scales in the problem might be.

\section{Smallest Events}

The smallest events are one block events having width $a$ and stress drop of $\sigma$. Since the compressional spring stiffness scales as $1 / a^{2}$, they move a distance of order $\sigma a^{2}$, and thus have a moment of order $\sigma a^{3}$. Calling the slip zone length of the smallest events $\hat{x}$ and moment $\hat{M}$, we have

$$
\hat{x}=a
$$

and

$$
\hat{M} \sim \sigma \hat{x}^{3} .
$$

These scales are seen in the numerics to set the size of the lower cutoff in the events. The distribution of the smallest events is seen to be a power law, showing a straight line on the $\ln R$ versus $w$ plots; this is easiest to see in Figure 3, where a smaller value of $a$ is shown, thereby extending the power law to smaller values of $M$. 


\section{Crossover Events}

The length $x^{\dagger}$, which is half the wavelength of the scale marking the crossover between marginally stable and unstable growth modes, occurs at, from (17),

$$
x^{\dagger}=\frac{\pi}{\sqrt{\alpha-1}}
$$

Estimating the average slip from the acceleration $\sigma$ times the square of the time of the event, with the time of the event being of order the length (since the sound speed has been normalized to unity), we get

$$
M^{\dagger} \sim \sigma x^{\dagger 3} .
$$

In Figure 6, the distribution of sizes of small events is plotted on a log-linear scale, with curves having different values of $\alpha$ shown. The straight lines on this plot evidence the exponential falloff of the distributions. An exponential falloff implies some scale; what is the scale? In Figure 7, the distributions are scaled by dividing the moments by $M^{\dagger}$ (with the overall constant in (23) set to unity). Here, on a $\ln R$ versus $M / M^{\dagger}$ plot, we see the lines appear parallel. So $M^{\dagger}$ seems to appropriately represent the $\alpha$ dependence.

\section{Delocalizing Events}

The crossover between small events and large events can be estimated by the length over which an exponentially growing slip pulse goes from having slip that scales with $\sigma$ to having slip that scales with 1 [Carlson and Langer, 1989]. Using the dispersion relation (17) to get a fastest growing rate of $e^{\sqrt{\alpha-1} t}$ gives a time (length) to go from slip $\sigma a^{2}$ to 1 of

$$
\tilde{x}=\frac{1}{\sqrt{\alpha-1}} \ln \frac{1}{\sigma a^{2}} \text {. }
$$

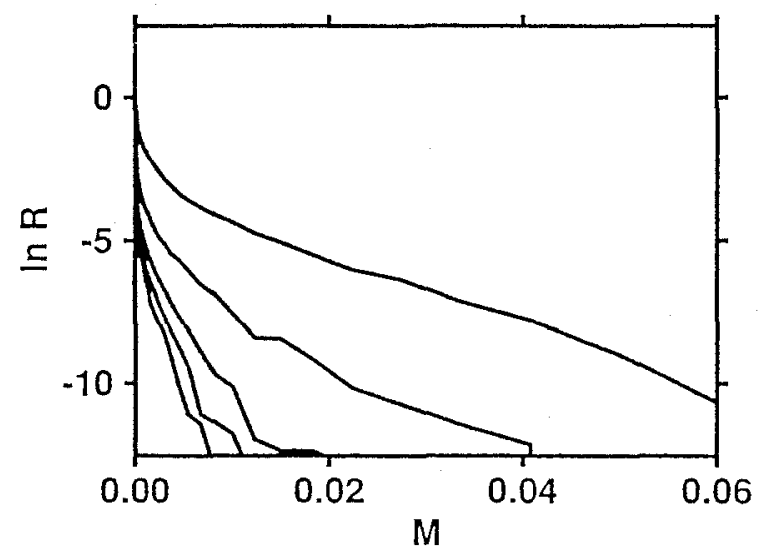

Figure 6. The exponential falloff of the distribution of small events is shown by plotting the logarithm of the rate $\ln R$ versus the moment $M$. The same curves shown in Figure 5 are used, except that only the larger values of $\alpha$ are used: $\alpha=4,6,8,10,12$ from top to bottom respectively. A straight line on this log-linear plot would be an exponential falloff.

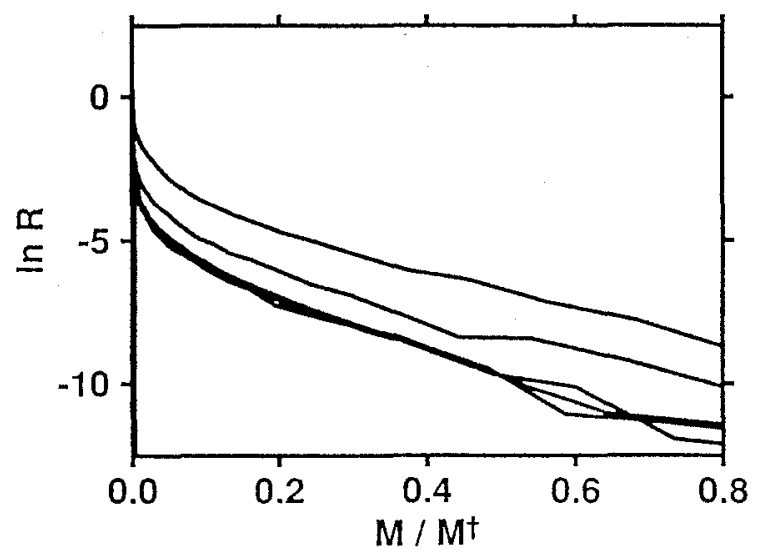

Figure 7. The dependence of the exponential decay on $\alpha$ is shown by a rescaling of the moments by $M^{\dagger}$ (see (23)). The same curves used in Figure 6 are used here. Note that the curves are parallel, showing that the $\alpha$ dependence in the exponential falloff has been scaled out.

Integrating the slip along this length gives

$$
\tilde{M} \sim \int_{0}^{\bar{x}} \sigma a^{2} e^{\sqrt{\alpha-1} x} d x \sim \frac{1}{\sqrt{\alpha-1}} .
$$

These scales are difficult to check, numerically, as an exponentially suppressed gap is seen to be opening up there in the slip-weakening distributions.

\section{Large Events}

For the largest events, the slip is of order unity, so the moment of the largest events $M^{*}$ scales witl the length of the largest events $x^{*}$ :

$$
M^{*} \approx x^{*} \text {. }
$$

Unfortunately, there's not much more we have to say about how to estimate what these values might be. Calculating the size of the largest events, in either the slipweakening case or the velocity-weakening case, remains an outstanding problem. Numerical results show that the largest events do not depend on either $a$ or $\sigma$, but do depend on $\alpha$. The large events account for almost all the net motion forward, and the peak of the largest events that dominate the motion,

$$
w^{*}=\ln M^{*}=\max \left(R(w) e^{w}\right)
$$

can be measured. Figure 8 shows a plot of this measured $M^{*}$ versus $\alpha$. For large values of $\alpha$, this shows a linear increase with $\alpha$, as the dotted line in Figure 8 indicates. Thus

$$
M^{*} \sim \alpha
$$

for large $\alpha$. We can also see in Figure 8 the transition to the small $\alpha$ regime, below $\alpha=1$. In the small $\alpha$ regime the biggest events scale with $\sigma$, unlike the large $\alpha$ regime where the large events are independent of $\sigma$. 


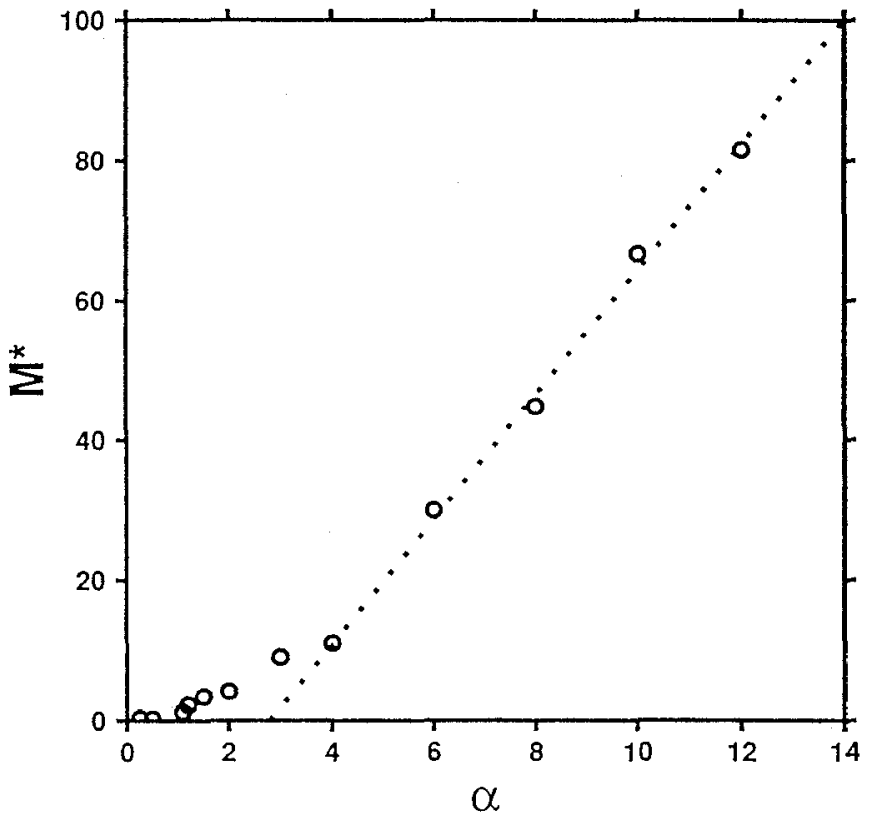

Figure 8. The dependence of the largest events on the slip-weakening $\alpha$. The moments $M^{*}$ of the events that dominate the net motion forward, as given by (27), are plotted for different values of the slip-weakening $\alpha$. The data used are the same as in Figure 5, along with a few smaller values of $\alpha$. Note the linear trend when $\alpha$ is big; the dotted line, which has an $\alpha$ intercept of 2.8 and a slope of 8.9 , shows this linear fit.

\section{Comparison with Velocity-Weakening Case}

The distributions of sizes of events for the slip-weakening and velocity-weakening frictions show some important similarities. In both, there is a transition from small values of weakening where there are only small events that scale with $\sigma$, to large values of weakening where there are small events that scale with $\sigma$ and large events that are independent of $\sigma$. The power law distribution of event sizes, for the differential rate of events $R$ having moments between $M$ and $M+d M$,

$$
R(M) \sim M^{-1-b}
$$

which is seen for the smallest events, has a value of $b=1$ for large values of $\alpha$. This exponent of $b=1$ for small events shows an astonishing universality across all of the dynamical models studied so far: for large weakening, it also holds for velocity weakening [Carlson and Langer, 1989], and in higher dimensional models as well [Carlson, 1991; Myers, Shaw, and Langer, preprint, 1994]. The origin of the exponent remains an outstanding unsolved problem of these dynamical models. Another similarity between the the slip- and velocity-weakening distributions is that there is a bump of large events which sticks up above the extrapolated small event rate; these large events dominate the net motion forward in the models.

There are two main differences between the distributions of sizes of events seen in the slip-weakening case as compared to the velocity-weakening case. The first concerns the transition from small events to large events. In the velocity-weakening case, the power law extended throughout the small event region. In contrast, in the slip-weakening case, we saw a crossover from a power law distribution to one that fell off exponentially at the upper end of the small event region (see Figures 6 and 7 ). Given that the origin of the power law has never been understood, it is not surprising that a finite cutoff to of a power law is also not understood. (More recent work on a two-dimensional elastodynamic model with long-range elastic interactions and slip-weakening friction, completed since this paper was originally submitted, shows the power law extending throughout the small event region, with no exponential cutoff [ Myers, Shaw, and Langer, preprint, 1994]).

The second major difference concerns the dependence of the large events on $\alpha$. In the slip-weakening case we saw that the large events continued to increase as $\alpha$ increased (Figure 8 ). In contrast, in the velocityweakening case the large events are seen to become asymptotically independent of $\alpha$ as $\alpha$ becomes large. This is all the more curious in the velocity-weakening case as the large events are seen to scale with $1 / \sqrt{\eta}$, when the viscous term is added to the dynamics [Shaw, 1994], and $1 / a$ when it is not [Carlson, et al., 1991]. The most obvious way that $\eta$ enters the velocity-weakening case with viscosity is through the smallest unstable lengthscale, which is $2 \pi \sqrt{\eta / \alpha}$; but since there is no dependence on $\alpha$, this can't be the way $\eta$ is entering as being relevant to the large events. There are interesting clues in all this regarding what is helping to create the large event complexity but, for now, no answers.

How does all this compare with data from real earthquake faults? Evidence from individual faults suggests that, at the end of a power law region [Gutenberg and Richter, 1954], there is a bump of large events [Wesnousky, et al., 1983; Singh, et al., 1983; Schwartz and Coppersmith, 1984; Davison and Scholz, 1985], which is consistent with the model. The exponent of the power law region of small events in the Earth has not been directly measured using moments, which would be the best test; empirical conversion formulas of magnitude to moment suggest, however, $a \cdot b$ value, in the language of (29) which would be less than 1 , more like $2 / 3$. If the data for the Earth really showed a $b$ value of $2 / 3$, for individual faults, and if the exponent for the models is really as universal as it appears to be at the present, if it continued to hold at $b=1$ for three dimensional tensorial elastodynamics, then that would be a serious problem for the models. In the absence of strong answers to both these two questions, however, it is premature to be overly concerned with this quantitative issue, when there appears to be reasonable qualitative consistency. A second issue is the distribution of events averaged over fault systems; data averaged over the whole Earth show two power law regions, with a bend at the crust depth [Pacheco, et al., 1992]. This depends, however, on what the distribution of faults is, about which this single-fault model has nothing to say. 


\section{Insensitivity to Cutoff in Frictional Weakening}

Having shown that the final value of the friction reduction is irrelevant, in that it can be scaled away (see (12) and (13) ), we turn now to the question of the effect that the nonlinear form of the cutoff has on the distribution of sizes of events. Here, we examine the three different forms of friction given by (13), (14), and (15). They all have the same forms of friction at low slip, starting with an initial drop of $\sigma$ and decreasing with slope $\alpha$, and the same saturation at high values of slip to the same constant value with zero slope, but differ in the way that they interpolate between these regimes, one having a polynomial form, one having an exponential form, and one being piecewise linear.

Examining the dependence on $\alpha$ in the three different forms, an interesting difference between small and large events is seen. For the small events, aside from an overall multiplicative constant in the rate, the distribution of event sizes is the same for the different frictional forms, for a given value of $\alpha$. In contrast, for the large events, there is an effective rescaling of $\alpha$ for the different functional forms. Figure 9 shows a plot of the dominant large event size $M^{*}$, as in (27), versus $\alpha$, for the three different functional forms. We see that, while all show a linear dependence of $M^{*}$ with $\alpha$, the constant relating $M^{*}$ to $\alpha$ varies between the functional forms. The polynomial form is seen to have the smallest effective $\alpha$, the exponential an intermediate effective $\alpha$, and the piecewise linear the largest effective $\alpha$. While the linear dependence of $M^{*}$ on $\alpha$ is not understood, we can at least see why the different functional forms

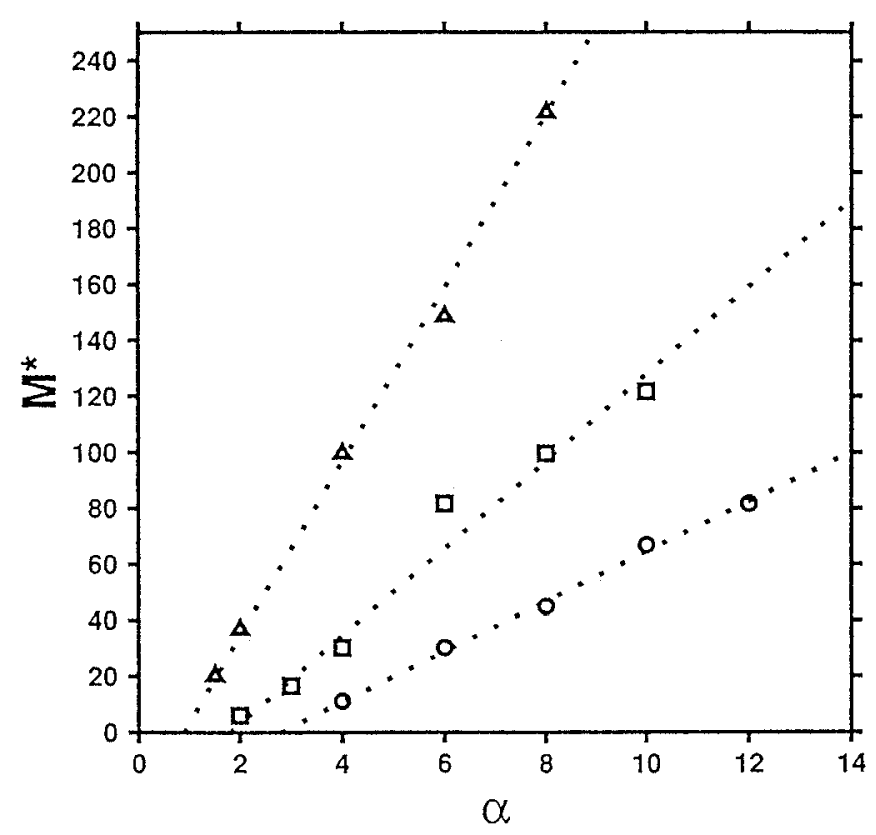

Figure 9. The dependence of the largest events $M^{*}$, as given by (27) on $\alpha$, for the three different functional forms of friction. The circles are the polynomial form, (13) the squares are the exponential form, (14) and the triangles are the piecewise linear form, (15). The dotted line linear fits to the three data sets for large $\alpha$ are $\alpha$ intercept 2.8 and slope $8.9, \alpha$ intercept 1.8 and slope 15.6 , and $\alpha$ intercept . 9 and slope 31.2 , respectively. would have the ordering that they do in the effective $\alpha$ that is exhibited; the large events extend out into the nonlinear regime, and the piecewise linear case has the least amount of friction beyond the linear regime, while the polynomial case has the most, of the three cases.

We have seen that the various nonlinear interpolations have minor and seemingly well-behaved effects on the behavior. And from the point of view of trying to model a physical process (such as, in this case, hydrofracturing) with some functional form, this relative insensitivity of the behavior to this aspect of the form is good news.

\section{Conclusion}

Slip-weakening friction, as well as velocity-weakening friction, has now been seen to produce slip complexity in simple dynamical models of earthquake faults. While this result has been examined here in a onedimensional model, it has also now been extended to a two-dimensional model which includes long-range elastic interactions, and slip complexity continues to be displayed there [ Myers, Shaw, and Langer, preprint, 1994]. The ability to obtain a well-behaved continuum limit in the slip-weakening case, without the need to introduce additional stabilizing terms and lengthscales, becomes even more significant in the higher dimensional models, where velocity weakening requires the introduction of additional stabilizing terms both along as well as perpendicular to the fault, to obtain proper continuum limits.

The derivation of the slip weakening and velocity weakening as two end-member cases of a physically plausible mechanism, and the demonstration that they both produce slip complexity, implies that the intermediate cases mixing slip and velocity weakening should also produce slip complexity. Thus slip complexity has been shown to be a generic feature of frictional weakening (sufficiently pronounced weakening, that is, as both cases required the weakening be larger than a critical value before intrinsic complexity was robustly produced).

The functional form of the saturation of the friction at high slip was examined and shown to not have any qualitative effect on the distribution of sizes of events but rather to have only well-controlled quantitative effects. One interesting difference in how the large and small events depended on the form of the friction at high slip was found: the large events were seen to depend on an effectively scaled weakening parameter, while the small events were seen to depend only on the bare unscaled weakening parameter. Nevertheless, the basic insensitivity in the results implies that the details of the way that the drop in friction may be limited by such processes as hydrofracturing are unimportant to the qualitative features of the behavior.

The results of this paper, together with the more recent results from the two-dimensional case which included long-range elastic interactions, enable, I believe, a strong assertion to be made: Frictional weakening in elastodynamic fault models gives slip complexity. The crucial ingredients for the complexity are frictional 
weakening on the fault, inertial dynamics, and the tying of the unsteady sliding layer to a stably sliding layer. These ingredients are also, I have argued, present in the Earth, with the coupling of the upper brittle crust to the lower ductile crust supplying the last ingredient above. The question then is this: what aspects of the complexity displayed by earthquakes come from the dynamical instabilities, and what aspects come from the fixed geometrical irregularities? Fortunately, the dynamical answer is a testable hypothesis, in that a variety of behaviors is implied from a minimal parametrization. We are working to develop higher dimensional models with tensor elasticity which will enable not only qualitative but quantitative testing of this dynamical hypothesis.

Acknowledgments. I benefited from discussions with Jim Langer and Chris Scholz. This work was supported by NSF grant 93-16513, USGS grant 1434-93-G-2284, and SCEC grant USC-572726.

\section{References}

Blanpied, M.L., T.E. Tullis, and J.D. Weeks, Frictional behavior of granite at low and high sliding velocities, Geophys. Res. Lett., 14, 554, 1987.

Blanpied, M.L., D.A. Lockner, and J.D. Byerlee, Fault stability inferred from granite sliding experiments at hydrothermal conditions, Geophys. Res. Lett., 18, 609, 1991.

Brace, W.F., and J.D. Byerlee, Stick-slip as a mechanism for earthquakes, Science, 153, 990, 1966.

Brace, W.F., and J.D. Byerlee, California earthquakes- Why only shallow focus?, Science, 168, 1573, 1970.

Brune, J.N., S. Brown, and P.A. Johnson, Rupture mechanism and interface separation in foam rubber models of earthquakes: a possible solution to the heat flow paradox and the paradox of large overthrusts, Tectonophysics, 218, $59,1993$.

Brune, J., T. Henyey, and R. Roy, Heat flow, stress, and rate of slip along the San Andreas Fault, California, J. Geophys. Res., 74, 3821, 1969.

Burridge, R., and L. Knopoff, Model and theoretical seismicity, Bull. Seis. Soc. Am., 57, 341, 1967.

Carlson, J.M., Two-dimensional model of a fault, Phys. Rev. A, 44, 6226, 1991.

Carlson, J.M., and J.S. Langer, Mechanical model of an earthquake fault, Phys. Rev. A, 40,6470, 1989.

Carlson, J.M., J.S. Langer, B.E. Shaw, and C. Tang, Intrinsic properties of a Burridge-Knopoff model of an earthquake fault, Phys. Rev. A, 44, 884-897, 1991.

Davison, F.C., and C.H. Scholz, Frequency-Moment Distribution of Earthquakes in the Aleutian Arc: A test of the Characteristic Earthquake Model, Bull. Seis. Soc. Am.; 75, 1349, 1985.

Dieterich, J.H., Time dependent friction and the mechanisms of stick-slip, Pure Appl. Geophys., 116, 790, 1978.

Dieterich, J.H., Modeling of rock friction: 1. Experimental results and constitutive equations, J. Geophys. Res., 84, $2161,1979$.

Gutenberg, B., and C.F. Richter, Seismicity of the Earth and Related Phenomena, Princeton Univ. Press, Princeton, N.J., 1954.

Horowitz, F.G., and A. Ruina, Slip patterns in a spatially homogeneous fault model, J. Geophys. Res., 94, 10,279, 1989.

Lachenbruch, A., Frictional heating, fluid pressure, and the resistance to fault motion, J. Geophys. Res., 85, 6097, 1980.
Lachenbruch, A., and J. Sass, Heat flow and energetics of the San Andreas Fault Zone, J. Geophys. Res., 85, 6185, 1980.

Langer, J.S., and H. Nakanishi, Models of crack propagation II: two dimensional model with dissipation on the fracture surface, Phys. Rev. E, 48, 439, 1993.

Langer, J.S., and C. Tang, Rupture propagation in a model of an earthquake fault, Phys. Rev. Lett., 67, 1043, 1991.

Mase, C.W., and L. Smith, Effects of frictional heating on the thermal, hydrologic, and mechanical response of a fault, J. Geophys. Res., 92, 6249, 1987.

McKenzie, D.P., and J.N. Brune, Melting on fault planes during large earthquakes, Geophys. J. R. Astron. Soc., $29,65,1972$.

Myers, C.R., and J.S. Langer, Rupture propagation, dynamical front selection, and the role of small length scales in a model of an earthquake fault, Phys. Rev. E, 47, 3048 1993.

Pacheco, J.F., C.H. Scholz, and L.R. Sykes, Changes in frequency size relationship from small to large earthquakes, Nature, 355, 71-3, 1992.

Pepke, S.L., J.M. Carlson, and B.E. Shaw, Prediction of large events on a dynamical model of a fault, J. Geophys. Res., 99, 6769, 1994.

Rice, J.R., Spatio-temporal Complexity of Slip on a Fault, J. Geophys. Res., 98, 9885, 1993.

Ruina, A., Slip instability and state variable friction laws, J. Geophys. Res., 88, 10359, 1983.

Scholz, C.H., P. Molnar, and T. Johnson, Detailed studies of frictional sliding of granite and implications for earthquake mechanisms J. Geophys. Res., 77, 6392, 1972.

Schwartz, D.P., and K.J. Coppersmith, Fault behavior and characteristic earthquakes: examples from the Wasatch and San Andreas fault zones, J. Geophys. Res. 89, 5681, 1984.

Shaw, B.E., Moment spectra in a simple model of an earthquake fault, Geophys. Res. Lett., 20, 643, 1993.

Shaw, B.E., Complexity in a spatially uniform continuum fault model, Geophys. Res. Lett., 21, 1983, 1994.

Shaw, B.E., J.M. Carlson, and J.S. Langer, Patterns of seismic activity preceding large earthquakes, J. Geophys. Res., 97, 479-88, 1992.

Sibson, R.H., Interactions between temperature and pore fluid pressure during earthquake faulting and a mechanism for partial or total stress relief, Nature, 243, 66, 1973.

Sibson, R.H., Generation of Pseudotachylyte by ancient seismic faulting, Geophys. J. R. Astron. Soc., 43, 775, 1975.

Sibson, R.H., Controls on low-stress hydro-fracture dilatancy in thrust, wrench and normal fault terrains, Nature, $289,665,1981$.

Singh, S., M. Rodriguez, and L. Esteva, Statistics of small earthquakes and frequency of occurrence of large earthquakes along the Mexican subduction zone, Bull. Seis. Soc. Am., 73, 1779, 1983.

Stesky R., W. Brace, D. Riley, and P-Y. Robin, Friction in faulted rock at high temperature and pressure, Tectonophysics, 23, 177, 1974.

Tse, S., and J.R. Rice, Crustal earthquake instability in relation to the depth variations of frictional slip properties, J. Geophys. Res., 91, 9452, 1986.

Wesnousky, S.G., C.H. Scholz, K. Shimazaki and T. Matsuda, Earthquakes frequency distribution and the mechanics of faulting, J. Geophys. Res., 88, 9331, 1983.

Bruce E. Shaw, Lamont-Doherty Earth Observatory, Columbia University, Palisades, NY 10964.

(Received July 19, 1994; revised March 27, 1995; accepted April 21, 1995.) 